\title{
O PAPEL DA EXPERIÊNCIA NO DESENVOLVIMENTO DE HABILIDADES DE DESIGN THINKER
}

\author{
The role of experience on the development of design thinker skills \\ FERRAZ, Mariana Garcia \\ Universidade Estadual de Londrina | UEL \\ marianagferraz@gmail.com
}

DEMARCHI, Ana Paula Perfetto

Universidade Estadual de Londrina | UEL

perfeto@sercomtel.com.br

\section{Resumo}

Esse projeto se dedica a compreender a influência da expertise no desenvolvimento das habilidades dos agentes de design. Baseando-se em entrevistas realizadas em cinco empresas de design da cidade de Londrina, foi possível identificar quais as técnicas e/ou métodos mais utilizados pelos profissionais e, assim, verificar como a Gestão Estratégica de Design e o Design Thinking intervêm no aperfeiçoamento das habilidades dos profissionais e no processo de inovação das empresas.

Palavras-Chave: Design thinking. Gestão estratégica de design. Habilidades de design thinker. Agentes de design.

\section{Abstract}

This study is dedicated on understanding the influence of expertise in developing the skills of design agents. Based on interviews conducted in five design companies in the city of Londrina, it was possible to identify which are the techniques and methods most used by professionals and, therefore, understand how Strategic Design Management and Design Thinking help improving their skills and how they support the innovation process of companies.

Keywords: Design thinking. Strategic design management. Design thinkers'skills. Design Agents. 


\section{INTRODUCÃO}

O design thinking trata de um processo que utiliza os métodos e abordagens do profissional do design a fim de gerar soluções eficientes e criativas para um determinado contexto, sendo também uma ferramenta fundamental para proporcionar inovação às empresas, tornando-as mais competitivas no mercado.

Diversos autores exploraram esse campo e deram suas próprias definições para o termo. Entre eles podemos destacar Brown (2009), Cross (2007), Lawson (2006) e Martin (2009).

Brown (apud MARTIN, 2009, p. 62) define o design thinking como

[...] uma disciplina que utiliza a sensibilidade e os métodos do designer para atender às necessidades das pessoas com o que é tecnologicamente fatível e qual estratégia viável de negócios pode converter valor para o cliente e oportunidades de mercado.

Para Martin (2009), significa pensar da mesma maneira com que um designer pensa e as organizações que adotam essa disciplina utilizam o processo designado por Pierce (1975), a razão abdutiva, a fim de formar hipóteses explicativas para os problemas de negócios, permitindo aos design thinkers explorar possibilidades futuras sem deixar de lado as alternativas averiguadas no passado.

Os autores Brown (2009), Cross (2007) e Martin (2009) compartilham o mesmo pensamento de que as habilidades de um design thinker são passíveis de serem desenvolvidas por meio da experiência. Além disso, os autores ressaltam quais as ferramentas imprescindíveis aos design thinkers, sendo algumas delas a observação, a imaginação, a habilidade de enxergar e resolver problemas mal definidos, a destreza em utilizar modelos gráficos/não verbais e a adoção de estratégias focadas diretamente na resolução dos problemas.

O design thinking possui caráter experimental. Por meio de briefings e das observações em campo, o designer consegue coletar os dados necessários para decifrar as necessidades e ambições de seu público-alvo. Após um longo período de análise, o designer deve interpretar os dados e sintetizar as ideias a fim de solucionar os problemas da melhor maneira possível.

Brown (2009) destaca que durante o processo, dois tipos de pensamentos são adotados, o divergente e o convergente. Durante o pensamento divergente deve-se criar o maior número de escolhas possíveis para um problema. Isto pode ser feito por meio de técnicas como o brainstorming, cujo "objetivo é abrir um amplo espectro de ideias. Outras abordagens são importantes para fazer escolhas, mas nada melhor que uma boa sessão de brainstorming para 
criar ideias. Já durante a fase convergente, devem-se eliminar as opções consideradas inapropriadas e decidir o melhor caminho a ser seguido.

De acordo com Martin (2009) o processo de conhecimento pessoal do design thinker é composto por três elementos principais que se influenciam de maneira mútua, a postura, as ferramentas e as experiências. Estes elementos, no entanto, não se manifestam em um processo rígido com fluxo único, podendo ser alterados e elaborados de forma que cada designer seja capaz de desenvolver suas próprias habilidades. $\mathrm{O}$ esquema pode ser visto na figura 1 abaixo.

Figura 1 - Sistema de conhecimento pessoal

\section{Seu sistema de conhecimento pessoal}

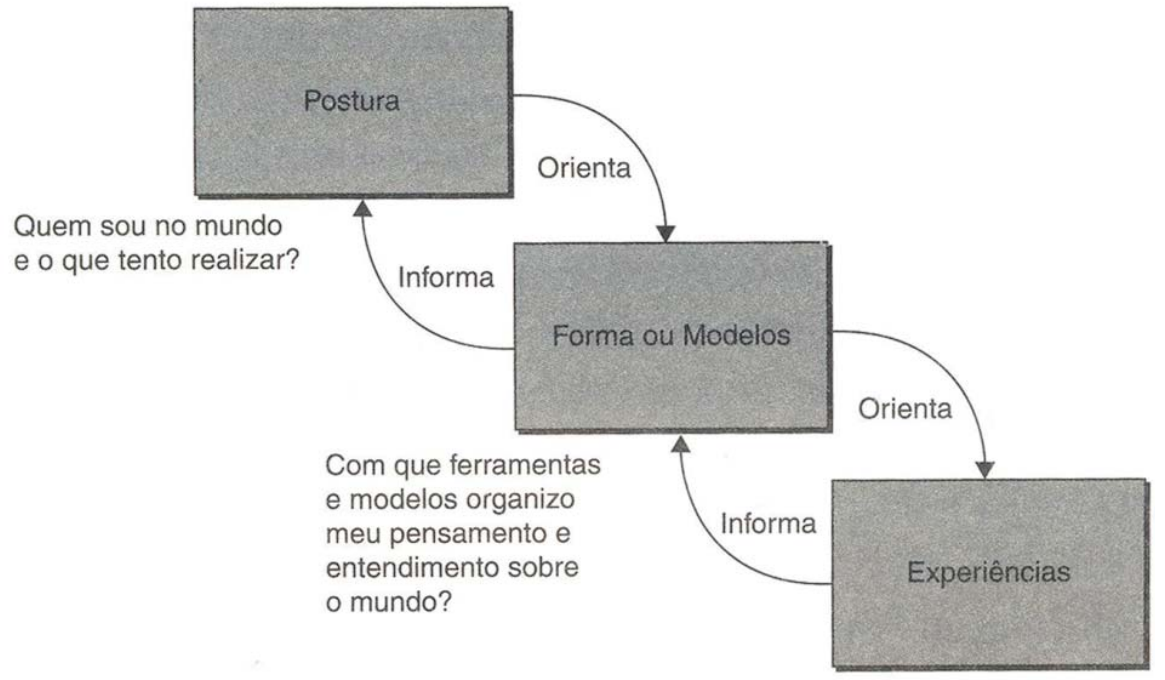

Com que experiências construo meu repertório de sensibilidades e habilidades?

Fonte: Martin (2009)

Sendo assim, tendo em vista os conceitos relacionados ao design thinking, esse projeto propõe-se a investigar, analisar e comparar as técnicas utilizadas por designers iniciantes e designers experientes, bem como as habilidades utilizadas por estes, a fim de mostrar os resultados e benefícios que esse processo pode oferecer, tanto para o profissional quanto para as empresas.

\section{MATERIAIS E MÉTODOS}

\section{Habilidades do design thinker no processo de Design Centrado no Usuário}

Os design thinkers possuem determinadas habilidades essenciais que os auxiliam durante o processo de compreensão e síntese de um problema, garantindo a elaboração de um bom projeto. 
Cross (2007) evidencia que entre essas habilidades estão a agilidade de pensamento, objetividade na tomada de decisões, facilidade de contextualização, além de curiosidade, imaginação e uma boa argumentação.

Para Lawson (2006), os design thinkers possuem capacidades narrativas assim como um contador de histórias, pois na contextualização de um projeto são hábeis em utilizar referências metafóricas, associações, analogias e mapas mentais. Isso os ajuda a acessar seus repertórios em busca de possíveis soluções que podem ser aplicadas para diversos contextos. Ou seja, por meio de uma solução encontrada, o designer acaba adquirindo conhecimento para solucionar problemas futuros.

Martin (2009, p. 160) destaca as ferramentas-chave dos design thinkers

[...] observação, imaginação e a configuração. [...] Uma vez que os design thinkers estão à procura de novos 'insights' que lhes permitam avançar o conhecimento, eles devem ser hábeis em ver coisas que outros não veem. Isto requer observar e ouvir cuidadosamente de maneira sensível ao assunto, como um etnógrafo faria.

Brown (2009 apud FORNASIER, 2011, p. 229), assim como Martin, também considera a observação uma habilidade primordial e afirma que

[...] os design thinkers observam como as pessoas se comportam; como o contexto das experiências os afetam nas relações com os produtos e serviços; como eles levam em conta o pensamento emocional e também sua performance funcional. A partir disso, tentam identificar o que as pessoas não declararam, ou coisas que sejam latentes, ou necessárias e transformam-nas em oportunidades.

Destaca ainda a empatia, o pensamento integrativo e multidisciplinar, o otimismo, a experimentação e a colaboração, sendo todas inerentes ao ser humano.

Em síntese, as habilidades determinantes em um design thinker são, entre outras, as habilidades de encontrar padrões; sintetizar as informações e conhecimentos extraídos; gerar novas ideias a partir de fragmentos, coleta de dados (etnografia), criatividade e imaginação; e gerar empatia.

Tendo em vista tais habilidades, o segundo passo é compreender como estas serão aplicadas para produzir um projeto de sucesso. Há uma série de 
abordagens utilizadas, no entanto, aqui, serão considerados aspectos relativos ao Processo de Design Centrado no Usuário.

No processo de Design Centrado no Usuário (DCU), o foco principal de um projeto desde o seu planejamento até a sua concepção - consiste em atender as necessidades e limitações dos usuários. Essa abordagem do design é considerada por muitos autores como a mais adequada, uma vez que a análise do comportamento e o entendimento das necessidades dos clientes levará à elaboração de projetos mais adequados e que atenda melhor a demanda de seus usuários.

Veryzer e Mozota (2005) caracterizam, por meio de um quadro comparativo, as diferenças entre a abordagem tradicional e a abordagem do Design Centrado no Usuário. Na abordagem tradicional a base é a tecnologia e o foco do projeto é no cliente atual e nas estratégias utilizadas. Já no Design Centrado no Usuário a base é o usuário, o estudo do cliente é feito por uma equipe multidisciplinar que focaliza em soluções que podem tanto ser úteis para clientes atuais quanto para clientes futuros, além disso, promove resultados que podem gerar competitividade e inovação.

Assim, pode-se constatar que as habilidades dos design thinkers auxiliam o Processo de Design Centrado no Usuário. O desenvolvimento de produtos inovadores por meio do cruzamento de informações entre profissionais de diferentes áreas (multidisciplinaridade) garante que a empresa consiga entender as necessidades dos diferentes tipos de usuários e, por meio das ferramentas visuais, consiga traduzir, efetivamente, essas necessidades em soluções para o cliente. A visualização e a criação de protótipos são as ferramentas mais valiosas para dar uma perspectiva holística aos projetos. (VERYZER; MOZOTA, 2005).

Esse processo de compreensão, síntese e criação do design thinker pode ser observado de maneira mais detalhada na figura 2 abaixo, retirada do modelo GEIDa (DEMARCHI; FORNASIER; MARTINS, 2014). 


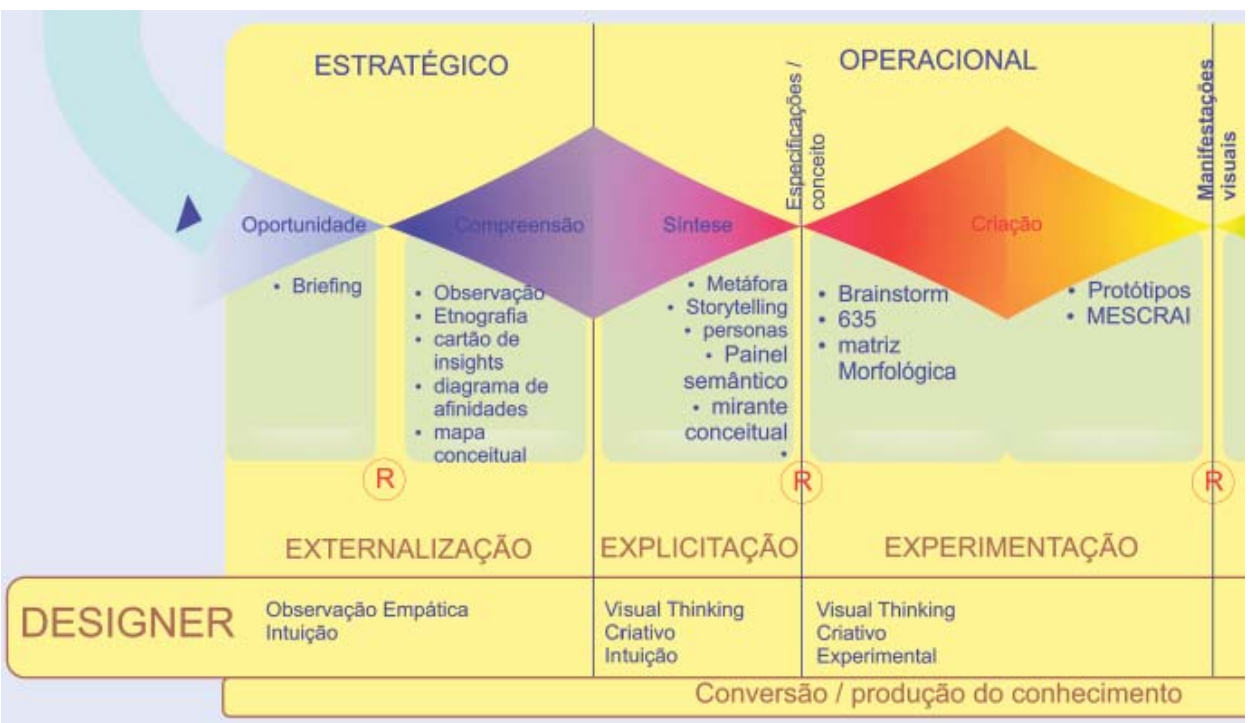

Fonte: Adaptado de Demarchi, Fornasier e Martins (2014).

O GEIDa é composto por quatro diamantes, que consistem em processos de divergência e convergência do pensamento do design thinker. Os dois primeiros ciclos, mostrados na figura 2, referem-se ao processo de análise, criação de estratégias e elaboração do projeto. Primeiramente, representado como a oportunidade, o agente de design realiza um briefing, onde investiga o propósito de seu cliente. Na compreensão o profissional observa de maneira minuciosa o contexto do projeto, tomando notas e criando painéis visuais que o ajudem a externalizar suas ideias. Durante a síntese, o agente de design, novamente com a ajuda de materiais gráficos, reúne as informações obtidas no processo, para, posteriormente, na etapa da criação, estender suas possibilidades e gerar ideias por meio de técnicas como o brainstorming, e assim conceber diferentes propostas gráficas. Finalmente, o agente de design, converge suas propostas, criando protótipos do produto final.

\section{METODOLOGIA}

A metodologia utilizada na pesquisa é de caráter etnográfico, com base em referencial teórico de Angrosino (2009). Trata-se de uma pesquisa qualitativa, onde o pesquisador analisará as práticas dos profissionais que utilizam o design thinking, a fim de evidenciar as características dos mesmos de acordo com o período de atuação no mercado.

O observador se posicionará de modo participante, se relacionando com os sujeitos da pesquisa sem interferir na coleta de dados. Terá ainda um cunho exploratório, pois contará com estudos bibliográficos e pesquisa de campo, por meio de observações e entrevistas, relacionando as ideias propostas pelos 
autores estudados com as experiências relatadas pelos profissionais.

Em relação à delimitação do campo de pesquisa foram selecionadas, primeiramente, 11 empresas de design. Posteriormente esse número foi reduzido para 05, tomando com base as metodologias utilizadas por tais empresas, uma vez que as mesmas empregam princípios específicos ou similares aos do design thinking, fato relevante para a temática do estudo. Foram escolhidas para a realização das entrevistas, respectivamente: Kalau Design, La Casa Comunicação, Eidee, Oapo Design e Visualitá.

Para a escolha foi considerado a diferença no tempo de mercado de cada uma das empresas, como pode ser observado no quadro abaixo.

Quadro 1 - Empresas visitadas

\begin{tabular}{|l|l|l|l|}
\hline Empresa & \multicolumn{1}{|c|}{$\begin{array}{c}\text { Nome } \\
\text { Kempo de } \\
\text { Mercado }\end{array}$} & $\begin{array}{c}\text { No de Agentes de } \\
\text { Design Entrevistados }\end{array}$ \\
\hline 02 & La Casa Comunicação & 13 anos & O1 (A) \\
\hline 03 & Eidee & 5 anos & O2 (B e C) E) \\
\hline 04 & Oapo Design & 10 anos & $01(F)$ \\
\hline 05 & Visualitá & 24 anos & $01(G)$ \\
\hline
\end{tabular}

Fonte: O próprio autor.

Analisando o quadro acima é possível verificar que a empresa mais nova é a Eidee, que possui 5 anos de mercado e encontra-se ainda incubada à universidade; Já a Visualitá é a mais antiga, com mais de 20 anos de mercado e uma vasta experiência na área.

Para as entrevistas foi previamente elaborado um roteiro contendo as perguntas-chave direcionadas aos agentes de design, onde foi possível identificar os métodos e técnicas utilizados pelos mesmos. O quadro 2, abaixo, apresenta o protocolo que permeou a observação participante. 
Quadro 2 - Protocolo

\begin{tabular}{|c|c|c|}
\hline Metodologia & Objetivo da Pesquisa & Perguntas Relevantes do Roteiro \\
\hline $\begin{array}{l}\text { Etnografia } \\
\text { Múltipla }\end{array}$ & $\begin{array}{l}\text { Estabelecer um parâmetro } \\
\text { entre o Design Thinking e a } \\
\text { dinâmica dos profissionais at- } \\
\text { uantes como design thinkers } \\
\text { de acordo com o período de } \\
\text { atuação no mercado. } \\
\text { Analisar as técnicas e } \\
\text { habilidades utilizadas pelos } \\
\text { extremos perfis de profission- } \\
\text { ais, o designer iniciante e o } \\
\text { designer experiente, a fim de } \\
\text { mostrar os resultados que o } \\
\text { processo pode oferecer. }\end{array}$ & $\begin{array}{l}\text { Utilizam técnicas durante o } \\
\text { desenvolvimento de um projeto? } \\
\text { Quais? } \\
\text { São utilizadas técnicas espe- } \\
\text { cíficas? (ferramentas visuais, } \\
\text { conceitos, protótipos, quadros, } \\
\text { mapas mentais, etc.) Alguma } \\
\text { delas é mais utilizada? } \\
\text { Você acredita que os métodos } \\
\text { ou técnicas utilizados influencia- } \\
\text { ram de maneira positiva nos re- } \\
\text { sultados do seu trabalho? Como? } \\
\text { Ao longo de sua experiência } \\
\text { profissional você modificou os } \\
\text { métodos utilizados no início da } \\
\text { carreira? Por quê? }\end{array}$ \\
\hline
\end{tabular}

Fonte: O próprio autor.

De todo o questionário quatro perguntas, que estão listadas no quadro 2 acima, se destacaram como essenciais para o objetivo dessa pesquisa. Essas questões consistiram, basicamente, em investigar se os profissionais utilizam técnicas e/ou métodos específicos durante a geração de alternativas e criação de seus projetos, identificá-las e relatar se as mesmas têm produzido resultados válidos. As respostas foram reunidas e analisadas, sendo organizadas em quadros informativos e comparativos.

\section{RESULTADOS E DISCUSSÃO}

Analisando os profissionais entrevistados pôde-se comprovar a efetividade dos estudos de diversos autores que abordam sobre o design thinking, entre eles os estudos de Brown (2009), que afirmam que as habilidades de um design thinker não estão restritas somente aos designers, podendo ser desenvolvidas por qualquer indivíduo por meio da experiência.

O quadro 3 abaixo mostra alguns dados extraídos das coletas de dados, entre eles a diversificação da formação acadêmica dos agentes de design, fato que comprova que a interdisciplinaridade é um fator determinante entre os profissionais que utilizam o design thinking. 
Quadro 3 - Dados dos agentes de design

\begin{tabular}{|c|c|c|c|}
\hline Empresa & $\begin{array}{l}\text { Agente de } \\
\text { Design }\end{array}$ & Formação Acadêmica & Anos de Experiência \\
\hline 01 & $A$ & $\begin{array}{l}\text { Administração; Espe- } \\
\text { cialização em Gestão } \\
\text { Estratégica de Design }\end{array}$ & $\begin{array}{l}20 \text { anos ( } 3 \text { com Gestão } \\
\text { Estratégica de Design) }\end{array}$ \\
\hline \multirow[t]{2}{*}{02} & B & $\begin{array}{l}\text { Design Gráfico; Especial- } \\
\text { ização em } \\
\text { Gestão Estratégica de } \\
\text { Design }\end{array}$ & 13 anos \\
\hline & C & $\begin{array}{l}\text { Publicidade e Propagan- } \\
\text { da }\end{array}$ & 11 anos \\
\hline \multirow[t]{2}{*}{03} & $D$ & $\begin{array}{l}\text { Desenho Industrial } \\
\text { (Design de Produto); Es- } \\
\text { pecialização em Gestão } \\
\text { de Design }\end{array}$ & 13 anos \\
\hline & $E$ & Design Gráfico & 5 anos \\
\hline 04 & $\mathrm{~F}$ & Design Gráfico & 10 anos \\
\hline 05 & G & Arquitetura & 29 anos \\
\hline
\end{tabular}

Fonte: O próprio autor.

O quadro 3 acima mostra ainda o tempo de experiência no mercado de trabalho de cada agente de design entrevistado, tanto nas respectivas empresas visitadas, quanto em outras empresas e/ou como freelancer.

O que se observou foi que os profissionais com menor experiência, como é o caso do agente $E$ que possui apenas 5 anos de mercado, ainda utilizam diversas técnicas na elaboração dos projetos. Já os agentes que estão há mais anos no mercado de trabalho, como o agente G , devido ao nível de expertise, acabam não se submetendo tanto as técnicas, não seguindo de maneira rigorosa as etapas do projeto.

Os agentes $D$ e $E$ relataram como é constituído o processo de desenvolvimento de projetos da empresa 03 (Eidee), que é em geral definido por quatro etapas: o briefing, a geração de ideias, a pré-visualização e por último a viabilidade de produção. Durante essas fases as principais técnicas utilizadas 
pelos agentes da empresa são pesquisas desk, mapas mentais, fluxogramas, painéis visuais, análises de similares, estudos volumétricos e criação de protótipos. Todas as etapas são muito bem definidas e o registro do processo é considerado essencial.

Foi possível identificar que, apesar do longo tempo de mercado do agente D (13 anos) e de seu grande conhecimento na área, principalmente na questão tecnológica relacionada ao design de produto, sentiu-se a necessidade de algum profissional que utilizasse algumas técnicas e/ou métodos capazes de externalizar as ideias e necessidades da empresa, e para isso, o agente de design $E$, se mostrou eficiente, levando inovação à empresa.

Já a agente $G$ da empresa 05 (Visualitá), declarou que as etapas dos projetos dependem muito da necessidade do cliente, não seguindo um planejamento tão regrado. As ferramentas visuais como esboços e sketches são sempre utilizadas e consideradas indispensáveis, no entanto, o processo criativo não possui um registro bem definido.

Os profissionais B e C da empresa O2 (La Casa Comunicação), apresentaram três etapas cruciais utilizadas pela agência: pesquisa, verificação e apresentação visual. No entanto, as etapas também não são bem definidas, podendo ser alteradas conforme a necessidade do cliente.

Segundo a descrição do agente $F$, a empresa 04 (Oapo Design), possui etapas e um processo bem semelhantes à empresa 02 , pois também não possui um procedimento fixo. $\mathrm{O}$ agente descreve que uma das ferramentas visuais mais utilizadas são os mockups, pois ajudam a visualizar o projeto final.

Oagente A , apesar de possuir aproximadamente 20 anos de experiência, teve contato com a Gestão Estratégica de Design e o Design Thinking há apenas 3 anos, então demonstra seguir de maneira mais estruturada as etapas do processo. Também faz grande uso da documentação, assim como o agente $E$.

A figura 3, abaixo, apresenta uma simples comparação entre as empresas, demonstrando quais se utilizam mais da intuição e quais são mais amparadas por técnicas. 


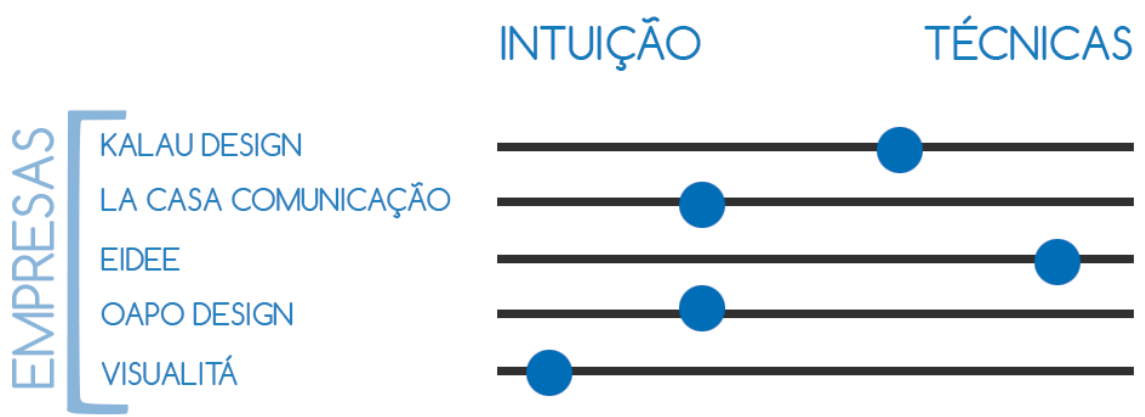

Em geral os profissionais que possuem expertise afirmam que a intuição acaba se tornando muito mais relevante no desenvolvimento dos projetos do que a própria técnica. Reafirmando a ideia de Martin (2009), de que com a experiência o agente de design aprimora sua sensibilidade, os agentes afirmam que à medida que novos problemas vão surgindo, tanto na geração de alternativas, quanto na viabilização, eles recorrem a soluções anteriormente utilizadas em outros projetos.

Constatou-se ainda que, apesar de os agentes trabalharem de formas distintas, alguns seguindo mais rigidamente as etapas do processo, e outros se atentando mais ao feeling, as ferramentas visuais são consideradas fundamentais e efetivas em todas as empresas visitadas. Em síntese, algumas ferramentas que são mais utilizadas, em geral: mapas mentais, sketches, painéis visuais e, principalmente, mockups.

\section{CONCLUSÕES}

Em síntese, pôde-se concluir por meio dessa pesquisa que, apesar de haver algumas técnicas e métodos considerados imprescindíveis pelos agentes de design entrevistados, como as ferramentas visuais, a experiência continua sendo, ainda, a habilidade mais explorada na hora de gerar alternativas e desenvolver um bom projeto final.

A parte positiva consiste na sensibilidade que a expertise garante aos profissionais, fazendo com que eles sejam capazes de identificar condições semelhantes em problemas passados para solucionar os problemas atuais. No entanto, pode também apresentar aspectos negativos, no que tange à inovação. Ao se prender à intuição, os agentes de design acabam deixando de lado algumas técnicas que poderiam auxiliar na busca de soluções mais criativas.

Portanto, para se tornar um bom design thinker e obter resultados 
satisfatórios, o profissional deve valorar as experiências adquiridas ao longo de sua carreira, sem deixar de lado o uso conjunto e equilibrado das técnicas e ferramentas oferecidas pelo design thinking.

\section{REFERÊNCIAS}

ANGROSINO, M. Etnografia e observação participante. Porto Alegre: Artmed, 2009.

BROWN, T. Change by design: how design thinking transforms organizations and inspires innovation. New York: HarperCollins, 2009. $264 \mathrm{p}$.

CROSS, N. Designerly ways of knowing. Basel: Birkhäuser Verlag Ag, 2007. $138 \mathrm{p}$.

DEMARCHI, A.; FORNASIER, C. B. R.; MARTINS, R. F. de F. Strategic integrator design management enhanced: a model based on the new knowledge management and design thinking. In: INTERNATIONAL FORUM OF DESIGN AS A PROCESS, 5., 2014, Guadalajara.

Proceedings... Guadalajara: Tecnológico de Monterrey, 2014. v. 1. p. 305-312.

\section{FORNASIER, C. B. R. Sistema de integração do conhecimento} organizacional pelo design thinker. 2011. $310 \mathrm{f}$. Tese (Doutorado em Engenharia e Gestão do Conhecimento) - Universidade Federal de Santa Catarina, Florianópolis, 2011.

LAWSON, B. How designers think: the design process demystified. 4. ed. Oxford: Elsevier, 2006. 321 p.

MARTIN, R. The design of business: why design thinking is the next competitive advantage. Boston: Harvard Business Press, 2009. 190 p.

VERYZER, R. W.; MOZOTA, B. B. The impact of user-oriented design on new product development: an examination of fundamental relationships. The Journal of Product Innovation Management, [S. I.], v. 22, n. 2, p. 128-143, Mar. 2005. 